\title{
Assessment of electron density profiles over the Brazilian region using radio occultation data aided by global ionospheric maps
}

(Preliminary results)

Gabriel Oliveira Jerez ${ }^{1,3}$, Fabricio dos Santos Prol ${ }^{2}$, Daniele Barroca Marra Alves ${ }^{1}$, João Francisco Galera Monico ${ }^{1}$ \& Manuel Hernandez-Pajares ${ }^{3}$

${ }^{1}$ Sao Paulo State University (UNESP), Cartography, Presidente Prudente, Brazil;

${ }^{2}$ German Aerospace Center (DLR), Neustrelitz, Germany;

${ }^{3}$ Universitat Politècnica de Catalunya (UPC-IonSAT), Barcelona, Spain.

\section{unesp ${ }^{\text {解 }}$}

UNIVERSIDADE ESTADUAL PAULISTA “JÚLIO DE MESQUITA FILHO"
UNIVERSITAT POLITÈCNICA DE CATALUNYA

BARCELONATECH 


\section{Method}

Assessment of radio occultation (RO) ionospheric profiles over the Brazilian area using ionosonde data aided by

Global Ionospheric Maps (GIM) information

Occultation position (Maximum electron density)

Ionosonde

Considering that the occultation occurrence is different from the ionosondes position, in order to compare RO data with ionosonde date, we used two approaches:

Assessment $\left\{\begin{array}{l}\text { Simple difference (RO - lonosonde) } \\ \text { Difference considering the variability of ionosphere from GIM (VTEC variability) }\end{array}\right.$

$$
\text { foF2 } 2 \text { ROtolONO }=\text { foF2_RO } \frac{\text { VTEC_IONO_GIM }}{\text { VTEC_RO_GIM }}
$$




\section{Data}

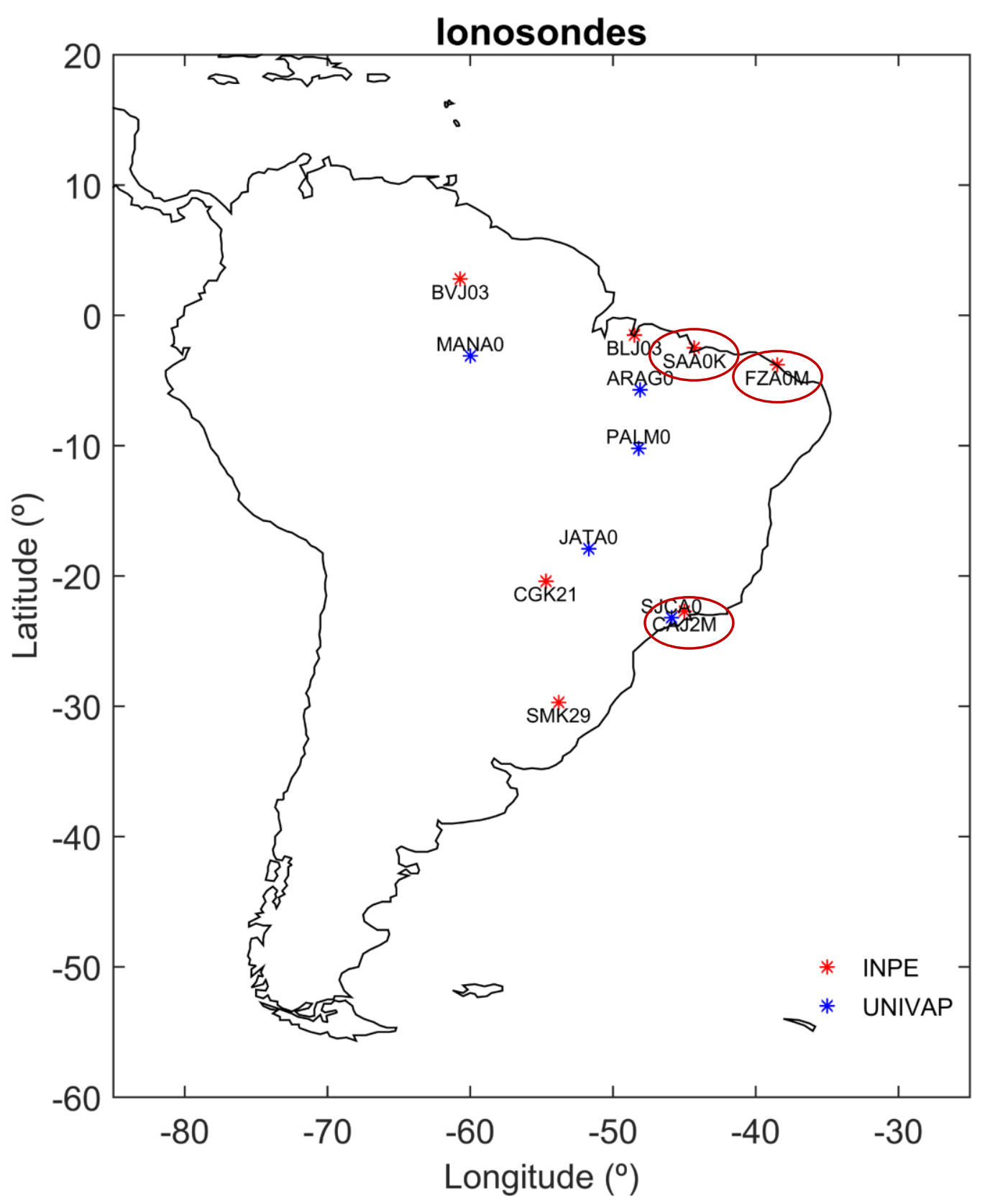

Doy 001-007/2012

RO data: COSMIC mission

GIMs: codg | corg | esag | esrg | igrg | igsg | jplg | upcg | uprg | whrg | whug | ehrg | uqrg

Search window: $20^{\circ} \times 20^{\circ}$ (lat $\times$ lon) 


\section{Results: CAJ2M}
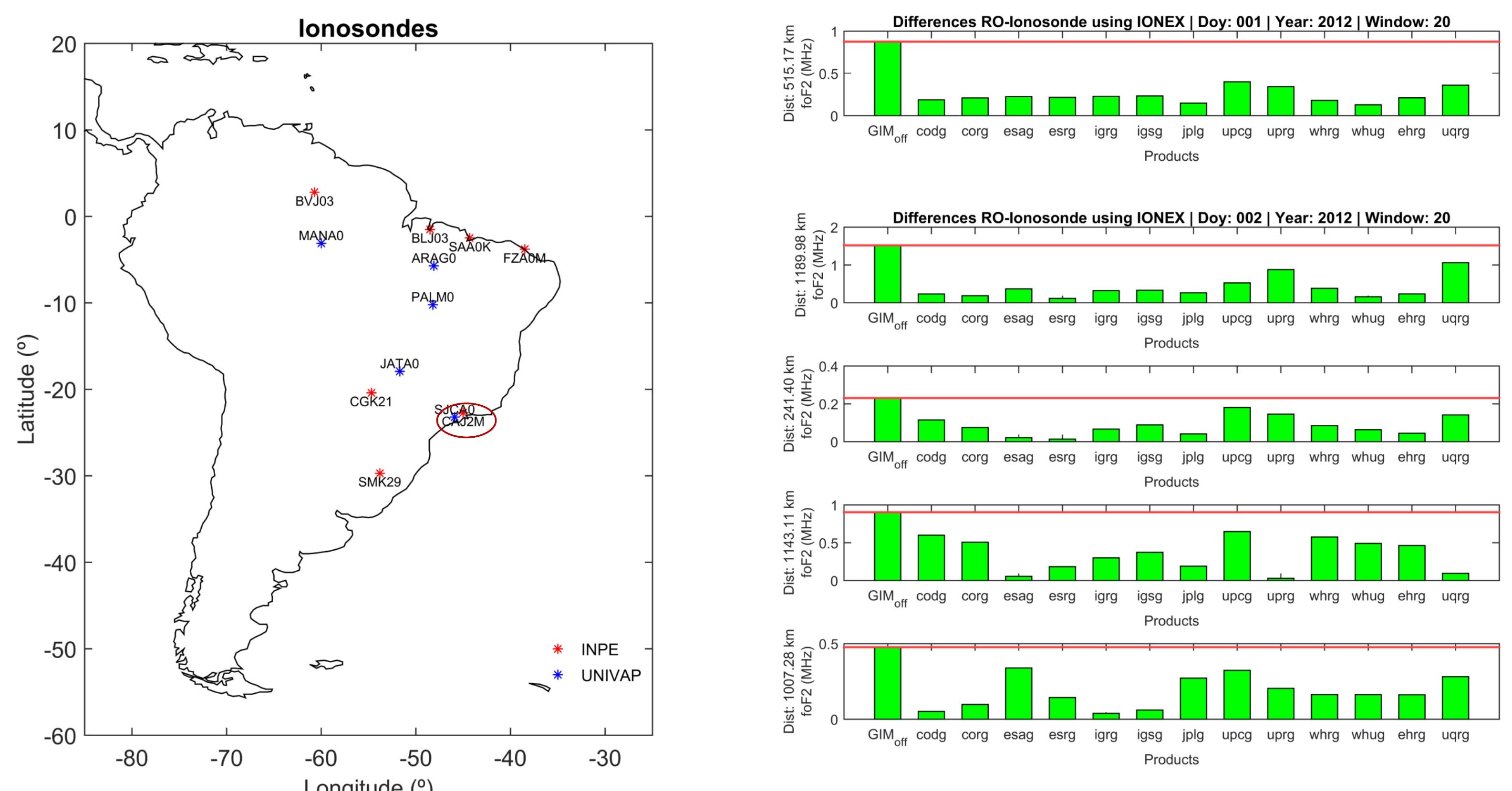


\section{Results: CAJ2M}
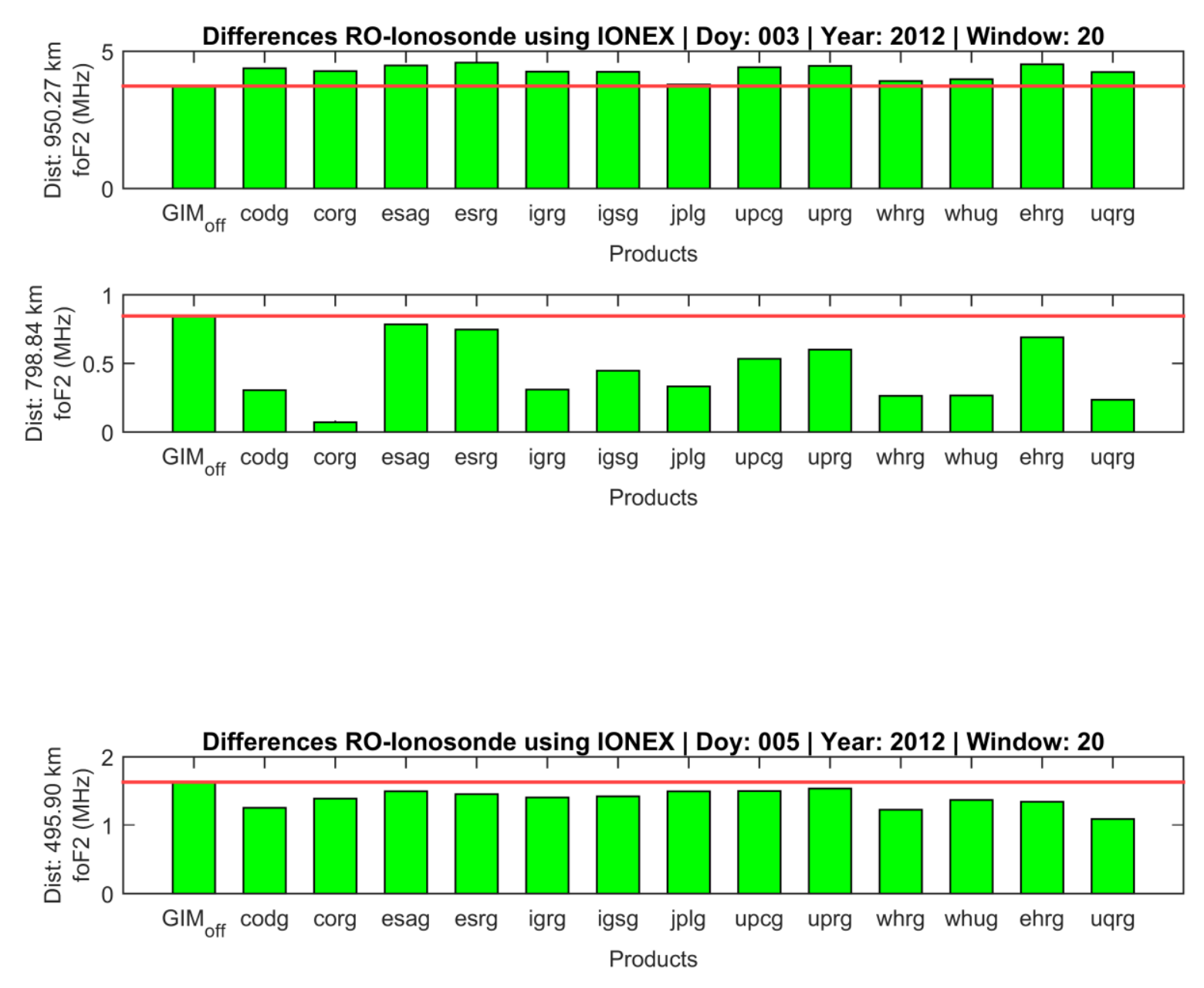
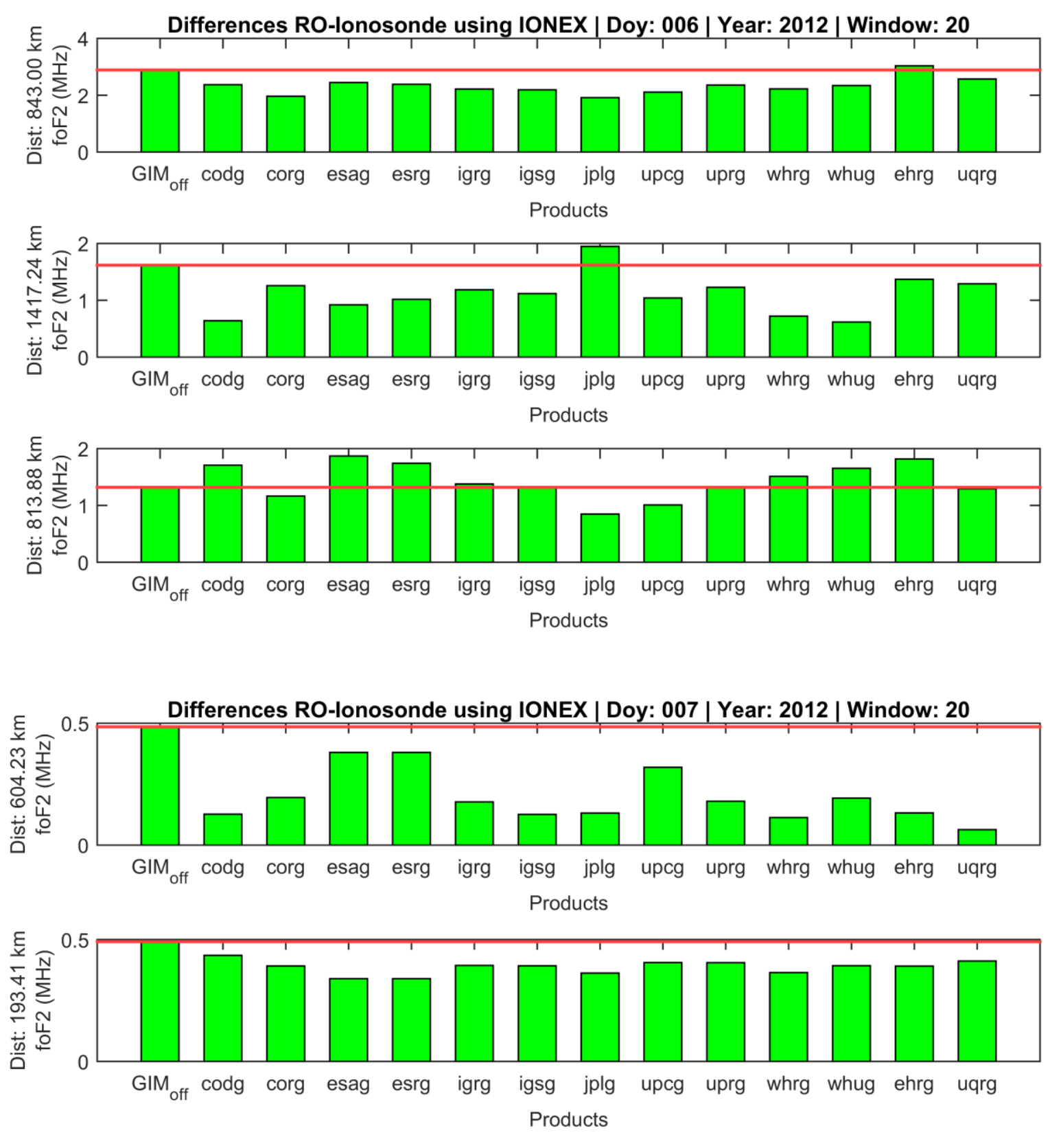


\section{Results: CAJ2M}

\section{Differences between RO and ionosonde data using GIMs}
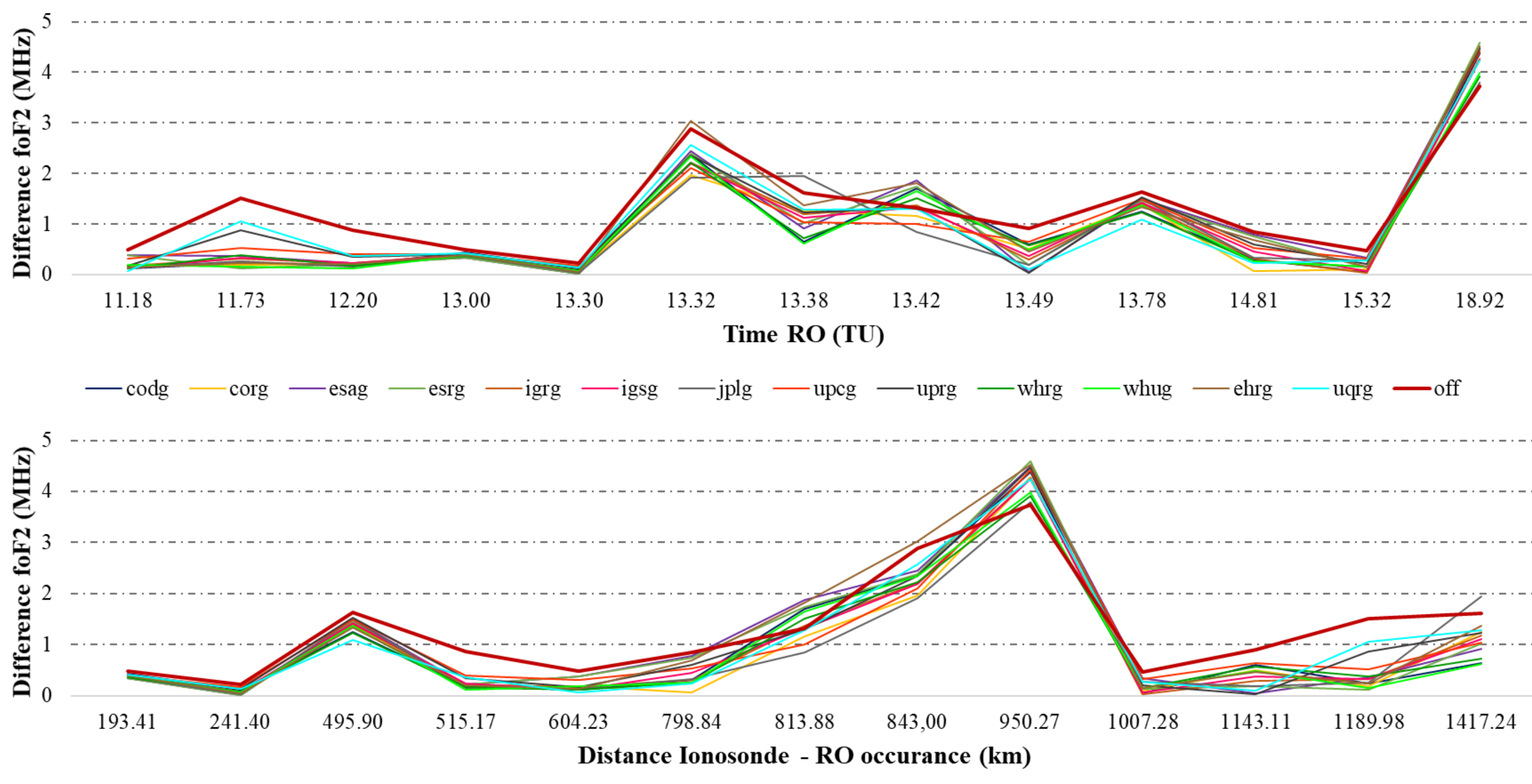

— codg - corg - esag - esrg - igrg -igsg - jplg - upcg - uprg - whrg - whug - ehrg - uqrg -off 


\section{Results: FZAOM}
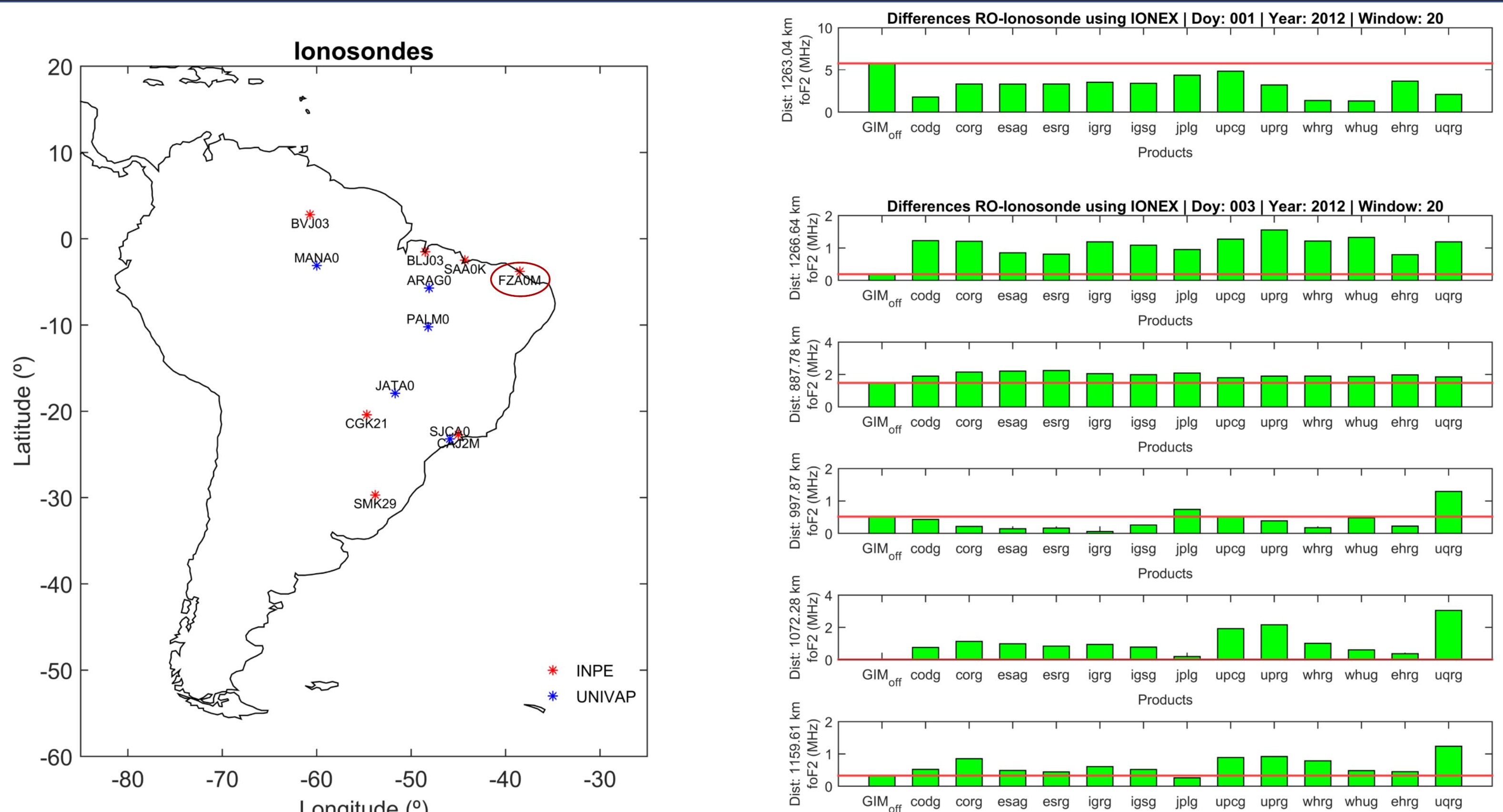


\section{Results: FZAOM}
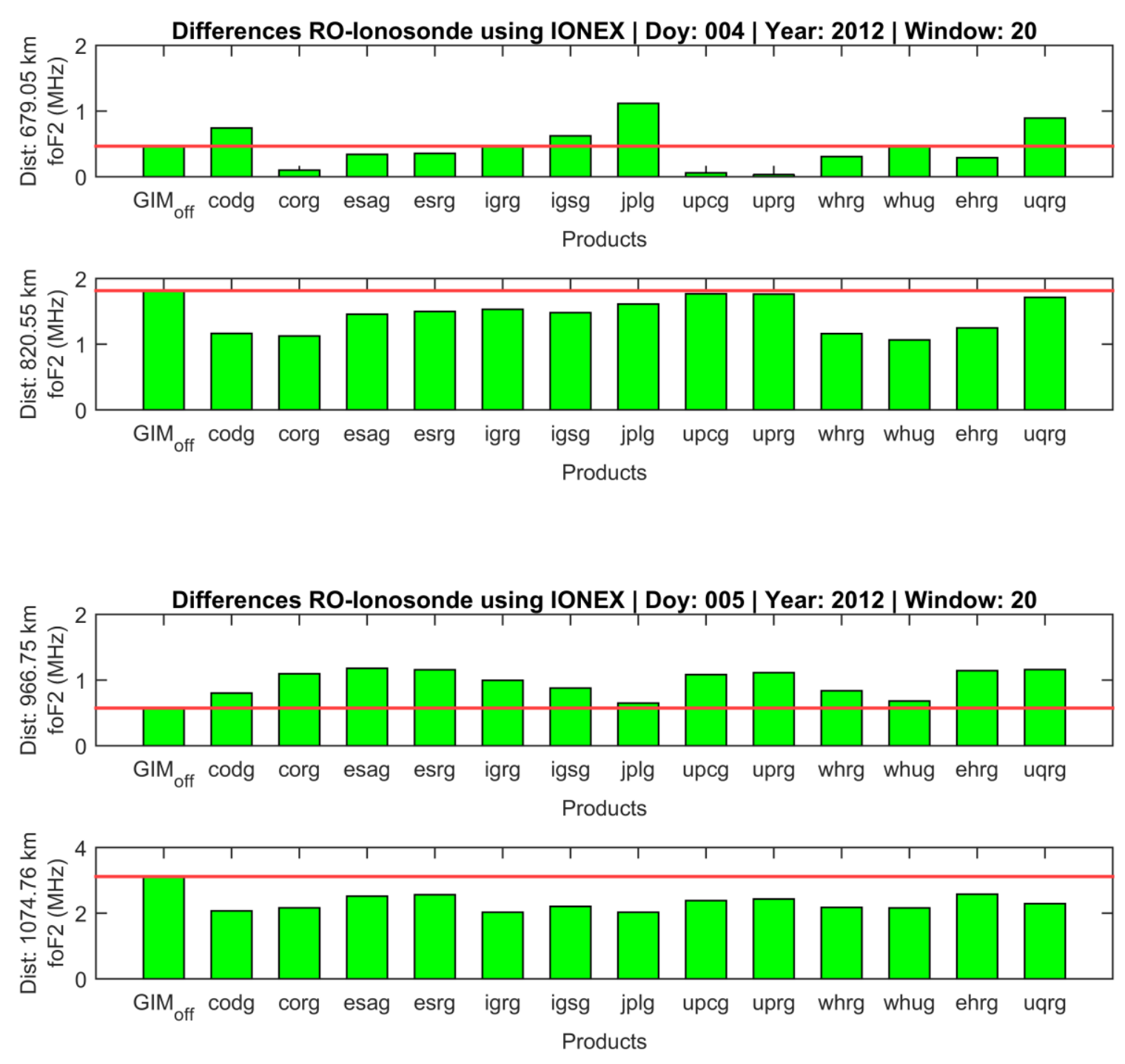
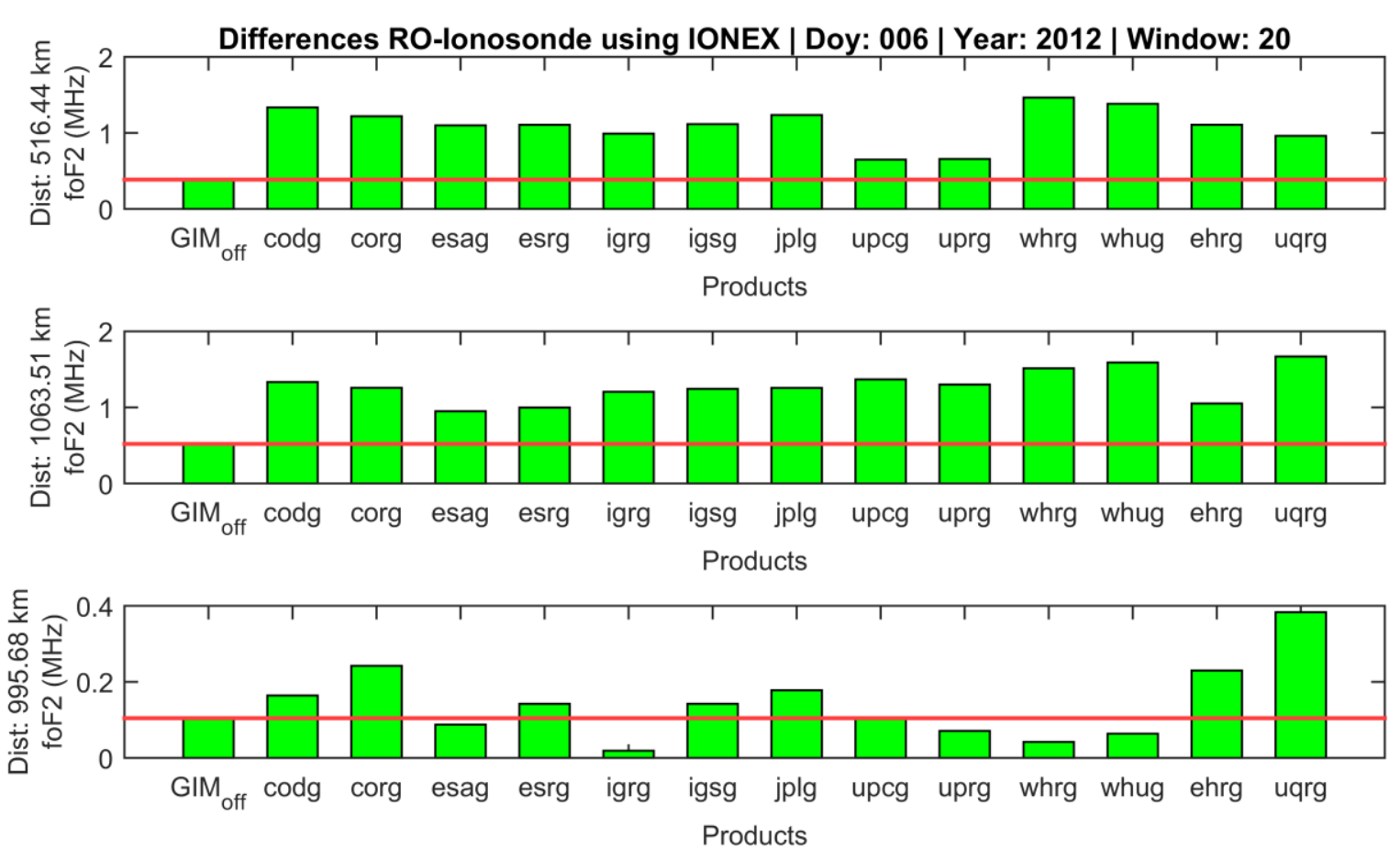


\section{Results: FAZOM}

Differences between RO and ionosonde data using GIMs
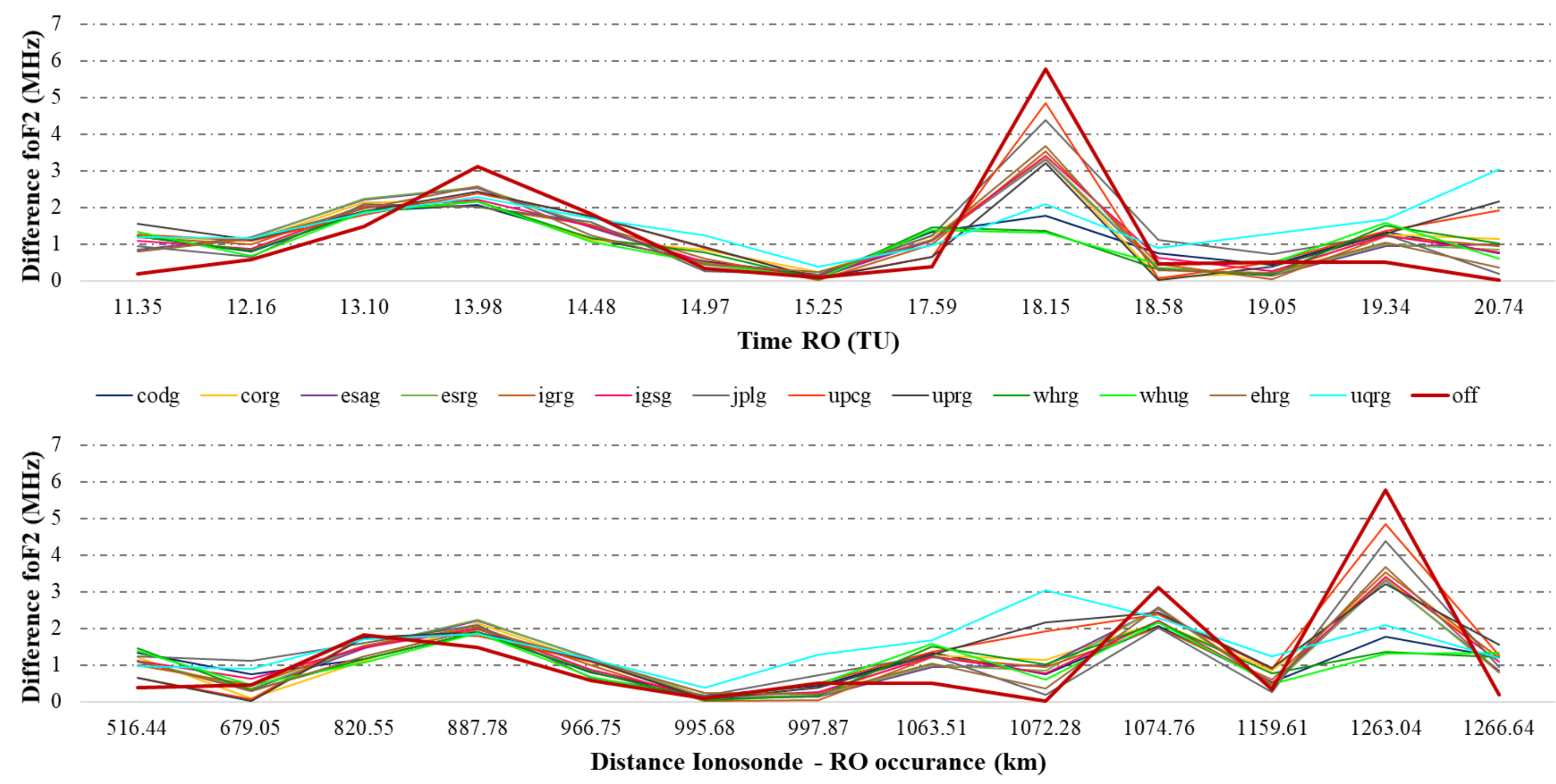


\section{Results: SAAOK}
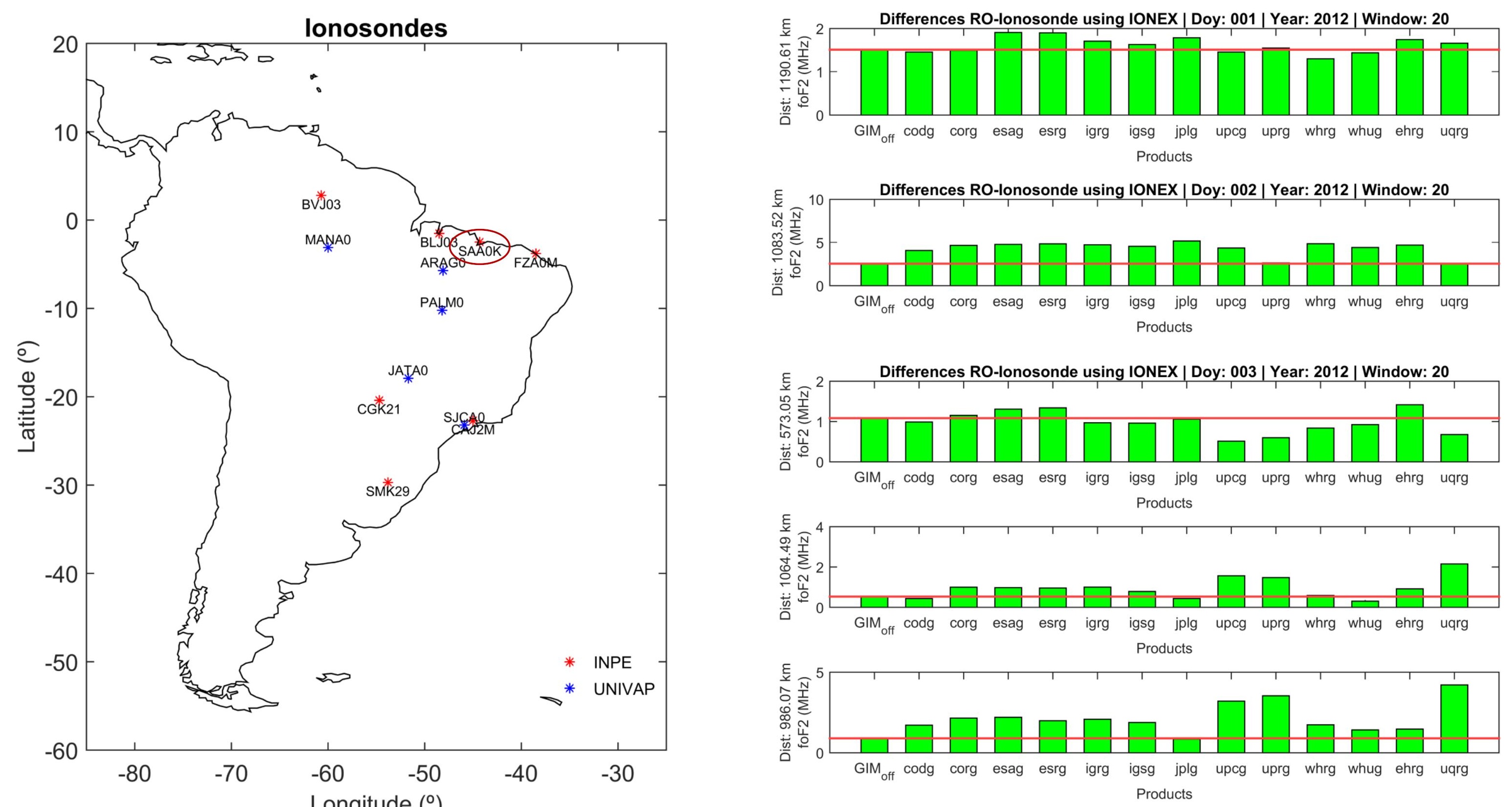


\section{Results: SAAOK}

Differences between RO and ionosonde data using GIMs
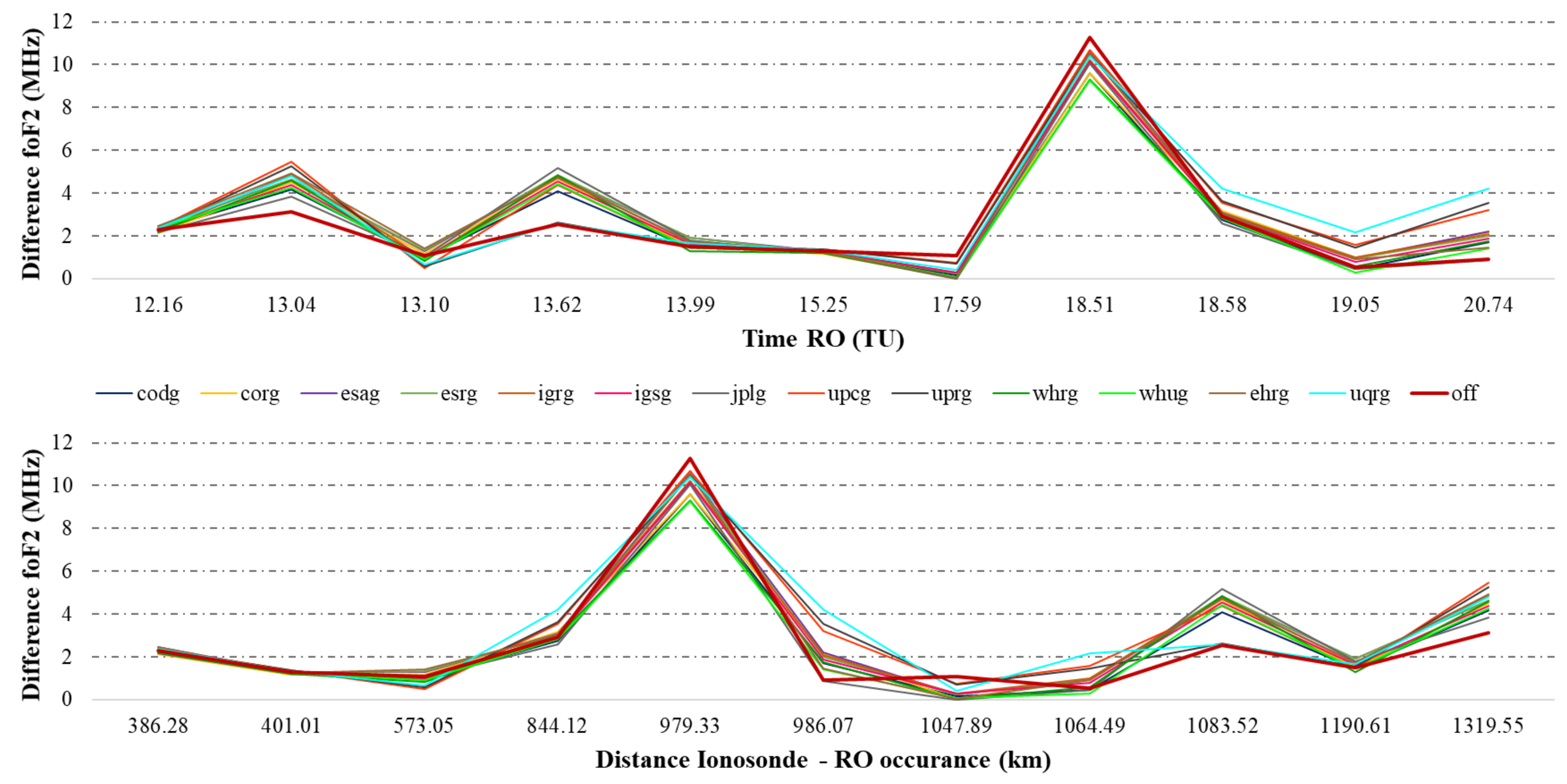

— codg - corg - esag - esrg - igrg - igsg - jplg - upcg - uprg - whrg - whug - ehrg - uqrg 


\section{Results: Summary}

\begin{tabular}{|c|c|c|c|c|c|c|c|c|c|c|c|c|c|c|c|c|c|c|}
\hline \multirow{2}{*}{ Ionosonde } & \multicolumn{18}{|c|}{ Differences foF2 ROtolONO by product (MHz) } \\
\hline & Doy & Time_iono & Time_ro & Distance & off & $\operatorname{codg}$ & corg & esag & esrg & igrg & igsg & jplg & upcg & uprg & whrg & whug & ehrg & uqrg \\
\hline \multirow{13}{*}{$\begin{array}{l}\underset{N}{\mathbb{S}} \\
\underset{\mathbb{S}}{\mathbf{S}}\end{array}$} & 1 & 12,17 & 12,20 & 515,17 & 0,876 & 0,188 & 0,210 & 0,226 & 0,216 & 0,228 & 0,233 & 0,148 & 0,400 & 0,344 & 0,180 & 0,129 & 0,211 & 0,361 \\
\hline & 2 & 11,67 & 11,73 & 1189,98 & 1,519 & 0,234 & 0,188 & 0,368 & 0,116 & 0,323 & 0,330 & 0,265 & 0,525 & 0,877 & 0,382 & 0,158 & 0,235 & 1,059 \\
\hline & 2 & 13,33 & 13,30 & 241,40 & 0,231 & 0,115 & 0,075 & 0,022 & 0,014 & 0,067 & 0,089 & 0,041 & 0,181 & 0,146 & 0,085 & 0,063 & 0,045 & 0,141 \\
\hline & 2 & 13,50 & 13,49 & 1143,11 & 0,904 & 0,601 & 0,508 & 0,057 & 0,184 & 0,301 & 0,374 & 0,191 & 0,648 & 0,030 & 0,576 & 0,492 & 0,463 & 0,096 \\
\hline & 2 & 15,33 & 15,32 & 1007,28 & 0,477 & 0,052 & 0,098 & 0,339 & 0,144 & 0,039 & 0,061 & 0,272 & 0,323 & 0,205 & 0,163 & 0,163 & 0,162 & 0,282 \\
\hline & 3 & 18,83 & 18,92 & 950,27 & 3,733 & 4,380 & 4,276 & 4,483 & 4,586 & 4,259 & 4,251 & 3,790 & 4,417 & 4,469 & 3,918 & 3,983 & 4,524 & 4,242 \\
\hline & 3 & 14,83 & 14,81 & 798,84 & 0,846 & 0,305 & 0,071 & 0,785 & 0,747 & 0,309 & 0,447 & 0,333 & 0,534 & 0,601 & 0,264 & 0,266 & 0,690 & 0,235 \\
\hline & 5 & 13,83 & 13,78 & 495,90 & 1,630 & 1,254 & 1,389 & 1,496 & 1,454 & 1,406 & 1,422 & 1,495 & 1,500 & 1,536 & 1,226 & 1,368 & 1,343 & 1,090 \\
\hline & 6 & 13,33 & 13,32 & 843,00 & 2,885 & 2,370 & 1,961 & 2,445 & 2,382 & 2,216 & 2,190 & 1,915 & 2,107 & 2,355 & 2,222 & 2,340 & 3,034 & 2,569 \\
\hline & 6 & 13,33 & 13,38 & 1417,24 & 1,618 & 0,640 & 1,257 & 0,921 & 1,016 & 1,185 & 1,118 & 1,949 & 1,041 & 1,228 & 0,722 & 0,618 & 1,369 & 1,289 \\
\hline & 6 & 13,50 & 13,42 & 813,88 & 1,318 & 1,707 & 1,164 & 1,869 & 1,742 & 1,376 & 1,328 & 0,847 & 1,007 & 1,310 & 1,511 & 1,653 & 1,817 & 1,292 \\
\hline & 7 & 11,17 & 11,18 & 604,23 & 0,485 & 0,127 & 0,195 & 0,380 & 0,380 & 0,177 & 0,126 & 0,131 & 0,319 & 0,180 & 0,113 & 0,193 & 0,132 & 0,064 \\
\hline & 7 & 13,00 & 13,00 & 193,41 & 0,491 & 0,435 & 0,392 & 0,340 & 0,340 & 0,394 & 0,392 & 0,362 & 0,406 & 0,405 & 0,365 & 0,393 & 0,391 & 0,412 \\
\hline \multirow{12}{*}{ 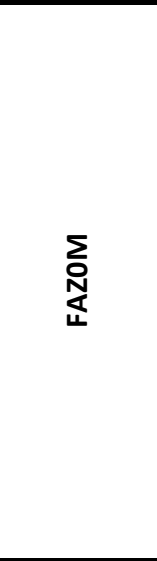 } & 1 & 18,17 & 18,15 & 1263,04 & 5,776 & 1,784 & 3,327 & 3,317 & 3,329 & 3,535 & 3,412 & 4,373 & 4,845 & 3,214 & 1,370 & 1,313 & 3,665 & 2,091 \\
\hline & 3 & 11,33 & 11,35 & 1266,64 & 0,189 & 1,226 & 1,206 & 0,850 & 0,810 & 1,188 & 1,086 & 0,951 & 1,273 & 1,556 & 1,214 & 1,326 & 0,792 & 1,188 \\
\hline & 3 & 13,17 & 13,10 & 887,78 & 1,484 & 1,899 & 2,148 & 2,208 & 2,244 & 2,050 & 1,989 & 2,089 & 1,801 & 1,900 & 1,903 & 1,874 & 1,977 & 1,850 \\
\hline & 3 & 19,00 & 19,05 & 997,87 & 0,518 & 0,431 & 0,216 & 0,143 & 0,163 & 0,058 & 0,258 & 0,740 & 0,511 & 0,387 & 0,176 & 0,483 & 0,224 & 1,295 \\
\hline & 3 & 20,67 & 20,74 & 1072,28 & 0,011 & 0,760 & 1,133 & 0,988 & 0,843 & 0,946 & 0,779 & 0,198 & 1,923 & 2,162 & 1,013 & 0,608 & 0,371 & 3,053 \\
\hline & 4 & 18,50 & 18,58 & 679,05 & 0,466 & 0,742 & 0,100 & 0,341 & 0,355 & 0,466 & 0,623 & 1,117 & 0,059 & 0,033 & 0,307 & 0,459 & 0,290 & 0,893 \\
\hline & 4 & 14,50 & 14,48 & 820,55 & 1,815 & 1,163 & 1,125 & 1,456 & 1,498 & 1,531 & 1,480 & 1,611 & 1,767 & 1,762 & 1,160 & 1,065 & 1,248 & 1,713 \\
\hline & 5 & 12,17 & 12,16 & 966,75 & 0,573 & 0,802 & 1,096 & 1,179 & 1,157 & 0,996 & 0,878 & 0,650 & 1,083 & 1,113 & 0,837 & 0,681 & 1,144 & 1,160 \\
\hline & 5 & 14,00 & 13,98 & 1074,76 & 3,116 & 2,071 & 2,163 & 2,519 & 2,562 & 2,028 & 2,206 & 2,027 & 2,384 & 2,431 & 2,176 & 2,160 & 2,578 & 2,291 \\
\hline & 6 & 17,67 & 17,59 & 516,44 & 0,386 & 1,335 & 1,219 & 1,099 & 1,107 & 0,991 & 1,115 & 1,236 & 0,649 & 0,658 & 1,464 & 1,383 & 1,107 & 0,962 \\
\hline & 6 & 19,33 & 19,34 & 1063,51 & 0,519 & 1,334 & 1,258 & 0,949 & 0,998 & 1,205 & 1,244 & 1,257 & 1,368 & 1,300 & 1,514 & 1,591 & 1,051 & 1,669 \\
\hline & 6 & 15,33 & 15,25 & 995,68 & 0,104 & 0,164 & 0,242 & 0,088 & 0,142 & 0,019 & 0,142 & 0,178 & 0,106 & 0,071 & 0,042 & 0,064 & 0,230 & 0,383 \\
\hline \multirow{11}{*}{ 㒸 } & 1 & 13,83 & 13,99 & 1190,61 & 1,511 & 1,455 & 1,496 & 1,908 & 1,900 & 1,707 & 1,630 & 1,786 & 1,455 & 1,548 & 1,299 & 1,439 & 1,744 & 1,660 \\
\hline & 2 & 13,67 & 13,62 & 1083,52 & 2,548 & 4,076 & 4,668 & 4,783 & 4,848 & 4,735 & 4,560 & 5,179 & 4,364 & 2,639 & 4,858 & 4,424 & 4,703 & 2,601 \\
\hline & 3 & 13,17 & 13,10 & 573,05 & 1,084 & 0,989 & 1,152 & 1,308 & 1,339 & 0,970 & 0,961 & 1,058 & 0,512 & 0,599 & 0,838 & 0,923 & 1,416 & 0,677 \\
\hline & 3 & 19,00 & 19,05 & 1064,49 & 0,532 & 0,435 & 0,999 & 0,976 & 0,959 & 1,002 & 0,787 & 0,435 & 1,566 & 1,477 & 0,590 & 0,305 & 0,917 & 2,152 \\
\hline & 3 & 20,67 & 20,74 & 986,07 & 0,899 & 1,715 & 2,153 & 2,203 & 1,989 & 2,080 & 1,880 & 0,855 & 3,204 & 3,537 & 1,736 & 1,420 & 1,470 & 4,211 \\
\hline & 4 & 18,50 & 18,51 & 979,33 & 11,267 & 9,616 & 9,602 & 10,562 & 10,538 & 10,214 & 10,147 & 10,060 & 10,699 & 10,521 & 9,290 & 9,278 & 10,512 & 10,381 \\
\hline & 4 & 18,50 & 18,58 & 844,12 & 2,909 & 2,763 & 3,150 & 3,070 & 3,054 & 3,059 & 2,947 & 2,571 & 3,545 & 3,619 & 2,949 & 2,839 & 2,991 & 4,211 \\
\hline & 5 & 12,17 & 12,16 & 386,28 & 2,273 & 2,296 & 2,105 & 2,442 & 2,423 & 2,206 & 2,296 & 2,174 & 2,359 & 2,342 & 2,229 & 2,368 & 2,466 & 2,363 \\
\hline & 6 & 17,67 & 17,59 & 1047,89 & 1,102 & 0,183 & 0,041 & 0,227 & 0,234 & 0,302 & 0,275 & 0,012 & 0,751 & 0,713 & 0,022 & 0,095 & 0,017 & 0,431 \\
\hline & 6 & 15,17 & 15,25 & 401,01 & 1,271 & 1,244 & 1,168 & 1,290 & 1,265 & 1,265 & 1,271 & 1,219 & 1,362 & 1,353 & 1,206 & 1,238 & 1,237 & 1,335 \\
\hline & 7 & 13,00 & 13,04 & 1319,55 & 3,147 & 4,151 & 4,511 & 4,638 & 4,638 & 4,893 & 4,388 & 3,817 & 5,454 & 5,246 & 4,581 & 4,258 & 4,939 & 4,797 \\
\hline
\end{tabular}




\section{Results: Summary}

\begin{tabular}{|c|c|c|c|c|c|c|c|c|c|c|c|c|c|c|c|c|c|c|}
\hline \multirow{2}{*}{ lonosonde } & \multicolumn{18}{|c|}{ Relative differences foF2 ROtolONO by product (\%) } \\
\hline & Doy & Time_iono & Time_ro & \begin{tabular}{|l|} 
Distance \\
\end{tabular} & off & codg & corg & esag & esrg & igrg & igsg & jplg & upcg & uprg & whrg & whug & ehrg & uqrg \\
\hline \multirow{13}{*}{ 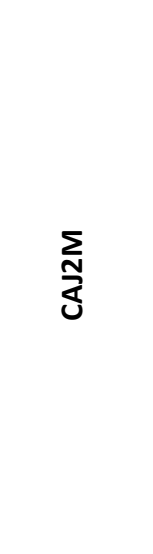 } & 1 & 12,17 & 12,20 & 515,17 & 9,62 & 2,06 & 2,30 & 2,48 & 2,38 & 2,50 & 2,56 & 1,63 & 4,40 & 3,78 & 1,98 & 1,41 & 2,32 & 3,96 \\
\hline & 2 & 11,67 & 11,73 & 1189,98 & 22,01 & 3,38 & 2,72 & 5,33 & 1,68 & 4,67 & 4,79 & 3,84 & 7,61 & 12,71 & 5,54 & 2,30 & 3,40 & 15,35 \\
\hline & 2 & 13,33 & 13,30 & 241,40 & 2,52 & 1,25 & 0,82 & 0,23 & 0,15 & 0,73 & 0,97 & 0,45 & 1,97 & 1,59 & 0,92 & 0,69 & 0,49 & 1,54 \\
\hline & 2 & 13,50 & 13,49 & 1143,11 & 9,82 & 6,53 & 5,52 & 0,61 & 2,00 & 3,27 & 4,06 & 2,07 & 7,04 & 0,33 & 6,26 & 5,34 & 5,03 & 1,04 \\
\hline & 2 & 15,33 & 15,32 & \begin{tabular}{|l|}
1007,28 \\
\end{tabular} & 4,17 & 0,45 & 0,86 & 2,97 & 1,26 & 0,34 & 0,53 & 2,38 & 2,83 & 1,79 & 1,43 & 1,43 & 1,42 & 2,46 \\
\hline & 3 & 18,83 & 18,92 & 950,27 & 27,55 & 32,32 & 31,56 & 33,09 & 33,85 & 31,43 & 31,37 & 27,97 & 32,60 & 32,98 & 28,91 & 29,40 & 33,39 & 31,31 \\
\hline & 3 & 14,83 & 14,81 & 798,84 & 7,62 & 2,75 & 0,64 & 7,07 & 6,73 & 2,79 & 4,03 & 3,00 & 4,81 & 5,41 & 2,38 & 2,40 & 6,22 & 2,12 \\
\hline & 5 & 13,83 & 13,78 & 495,90 & 18,52 & 14,25 & 15,79 & 17,00 & 16,52 & 15,98 & 16,16 & 16,99 & 17,04 & 17,45 & 13,93 & 15,55 & 15,26 & 12,39 \\
\hline & 6 & 13,33 & 13,32 & 843,00 & 33,45 & 27,47 & 22,74 & 28,34 & 27,62 & 25,69 & 25,39 & 22,20 & 24,43 & 27,31 & 25,76 & 27,13 & 35,18 & 29,78 \\
\hline & 6 & 13,33 & 13,38 & \begin{tabular}{|l|}
1417,24 \\
\end{tabular} & 18,76 & 7,42 & 14,57 & 10,67 & 11,78 & 13,74 & 12,97 & 22,59 & 12,07 & 14,24 & 8,37 & 7,17 & 15,87 & 14,95 \\
\hline & 6 & 13,50 & 13,42 & 813,88 & 14,02 & 18,16 & 12,38 & 19,88 & 18,53 & 14,64 & 14,12 & 9,01 & 10,72 & 13,94 & 16,07 & 17,59 & 19,33 & 13,75 \\
\hline & 7 & 11,17 & 11,18 & 604,23 & 5,14 & 1,34 & 2,07 & 4,02 & 4,02 & 1,88 & 1,33 & 1,39 & 3,38 & 1,90 & 1,20 & 2,04 & 1,40 & 0,67 \\
\hline & 7 & 13,00 & 13,00 & 193,41 & 5,14 & 4,56 & 4,10 & 3,56 & 3,56 & 4,12 & 4,11 & 3,80 & 4,25 & 4,24 & 3,82 & 4,11 & 4,09 & 4,31 \\
\hline \multirow{13}{*}{ 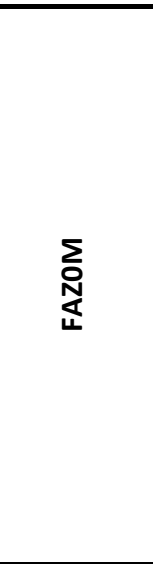 } & 1 & 18,17 & 18,15 & \begin{tabular}{|l|}
1263,04 \\
\end{tabular} & 70,44 & 21,76 & 40,57 & 40,45 & 40,60 & 43,11 & 41,60 & 53,32 & 59,08 & 39,20 & 16,70 & 16,01 & 44,70 & 25,50 \\
\hline & 3 & 11,33 & 11,35 & 1266,64 & 2,21 & 14,34 & 14,10 & 9,95 & 9,47 & 13,90 & 12,70 & 11,12 & 14,89 & 18,20 & 14,20 & 15,50 & 9,27 & 13,90 \\
\hline & 3 & 13,17 & 13,10 & 887,78 & 18,78 & 24,04 & 27,19 & 27,95 & 28,41 & 25,95 & 25,18 & 26,44 & 22,80 & 24,05 & 24,08 & 23,72 & 25,02 & 23,41 \\
\hline & 3 & 19,00 & 19,05 & 997,87 & 5,54 & 4,61 & 2,31 & 1,53 & 1,74 & 0,62 & 2,75 & 7,92 & 5,47 & 4,14 & 1,88 & 5,17 & 2,40 & 13,85 \\
\hline & 3 & 20,67 & 20,74 & \begin{tabular}{|l|}
1072,28 \\
\end{tabular} & 0,11 & 7,71 & 11,50 & 10,03 & 8,56 & 9,61 & 7,91 & 2,01 & 19,52 & 21,95 & 10,29 & 6,18 & 3,77 & 30,99 \\
\hline & 3 & 15,00 & 14,97 & 1159,61 & 3,81 & 6,06 & 9,91 & 5,69 & 5,15 & 7,07 & 6,03 & 3,02 & 10,33 & 10,70 & 9,14 & 5,62 & 5,24 & 14,39 \\
\hline & 4 & 18,50 & 18,58 & 679,05 & 4,88 & 7,77 & 1,05 & 3,57 & 3,72 & 4,87 & 6,52 & 11,70 & 0,62 & 0,35 & 3,22 & 4,80 & 3,03 & 9,35 \\
\hline & 4 & 14,50 & 14,48 & 820,55 & 24,36 & 15,61 & 15,10 & 19,55 & 20,11 & 20,55 & 19,86 & 21,62 & 23,72 & 23,65 & 15,58 & 14,30 & 16,75 & 23,00 \\
\hline & 5 & 12,17 & 12,16 & 966,75 & 7,70 & 10,77 & 14,71 & 15,83 & 15,53 & 13,37 & 11,78 & 8,72 & 14,54 & 14,94 & 11,23 & 9,14 & 15,35 & 15,56 \\
\hline & 5 & 14,00 & 13,98 & \begin{tabular}{|l|}
1074,76 \\
\end{tabular} & 43,28 & 28,76 & 30,04 & 34,98 & 35,58 & 28,16 & 30,63 & 28,16 & 33,11 & 33,76 & 30,22 & 30,00 & 35,81 & 31,81 \\
\hline & 6 & 17,67 & 17,59 & 516,44 & 3,68 & 12,72 & 11,61 & 10,47 & 10,54 & 9,43 & 10,62 & 11,77 & 6,18 & 6,26 & 13,94 & 13,17 & 10,55 & 9,16 \\
\hline & 6 & 19,33 & 19,34 & \begin{tabular}{|l|}
1063,51 \\
\end{tabular} & 5,38 & 13,82 & 13,03 & 9,84 & 10,34 & 12,48 & 12,89 & 13,02 & 14,18 & 13,48 & 15,69 & 16,48 & 10,89 & 17,30 \\
\hline & 6 & 15,33 & 15,25 & 995,68 & 1,23 & 1,93 & 2,85 & 1,03 & 1,67 & 0,22 & 1,67 & 2,09 & 1,25 & 0,83 & 0,50 & 0,75 & 2,70 & 4,51 \\
\hline \multirow{11}{*}{ 率 } & 1 & 13,83 & 13,99 & \begin{tabular}{|l|}
1190,61 \\
\end{tabular} & 20,15 & 19,40 & 19,94 & 25,44 & 25,33 & 22,76 & 21,73 & 23,82 & 19,40 & 20,64 & 17,32 & 19,18 & 23,25 & 22,13 \\
\hline & 2 & 13,67 & 13,62 & 1083,52 & 32,88 & 52,60 & 60,24 & 61,71 & 62,55 & 61,10 & 58,83 & 66,82 & 56,30 & 34,05 & 62,69 & 57,08 & 60,68 & 33,56 \\
\hline & 3 & 13,17 & 13,10 & 573,05 & 13,05 & 11,92 & 13,88 & 15,75 & 16,14 & 11,68 & 11,57 & 12,74 & 6,17 & 7,21 & 10,10 & 11,12 & 17,06 & 8,15 \\
\hline & 3 & 19,00 & 19,05 & 1064,49 & 6,41 & 5,24 & 12,03 & 11,76 & 11,55 & 12,07 & 9,48 & 5,24 & 18,87 & 17,79 & 7,11 & 3,67 & 11,04 & 25,92 \\
\hline & 3 & 20,67 & 20,74 & 986,07 & 10,03 & 19,13 & 24,02 & 24,58 & 22,20 & 23,21 & 20,97 & 9,54 & 35,75 & 39,46 & 19,37 & 15,84 & 16,41 & 46,98 \\
\hline & 4 & 18,50 & 18,51 & 979,33 & 182,46 & 155,73 & 155,49 & 171,04 & 170,65 & 165,40 & 164,32 & 162,91 & 173,26 & 170,37 & 150,44 & 150,24 & 170,23 & 168,12 \\
\hline & 4 & 18,50 & 18,58 & 844,12 & 47,11 & 44,75 & 51,01 & 49,72 & 49,46 & 49,54 & 47,73 & 41,63 & 57,40 & 58,61 & 47,76 & 45,97 & 48,44 & 68,20 \\
\hline & 5 & 12,17 & 12,16 & 386,28 & 39,54 & 39,93 & 36,62 & 42,47 & 42,14 & 38,37 & 39,94 & 37,81 & 41,03 & 40,74 & 38,77 & 41,18 & 42,88 & 41,09 \\
\hline & 6 & 17,67 & 17,59 & \begin{tabular}{|l|}
1047,89 \\
\end{tabular} & 12,23 & 2,03 & 0,46 & 2,52 & 2,60 & 3,35 & 3,05 & 0,14 & 8,33 & 7,91 & 0,24 & 1,06 & 0,19 & 4,78 \\
\hline & 6 & 15,17 & 15,25 & 401,01 & 17,83 & 17,46 & 16,40 & 18,10 & 17,75 & 17,75 & 17,84 & 17,10 & 19,11 & 18,99 & 16,92 & 17,37 & 17,37 & 18,73 \\
\hline & 7 & 13,00 & 13,04 & 1319,55 & 35,12 & 5,32 & 50,34 & 51,75 & 51,75 & 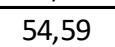 & 48,96 & 42,59 & 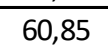 & 8,54 & 51,12 & 47,51 & 55,11 & 53,53 \\
\hline
\end{tabular}




\section{Results}

Some results did not present the same pattern, considering distance or region. Some of them could be explained by the ionospheric variability.
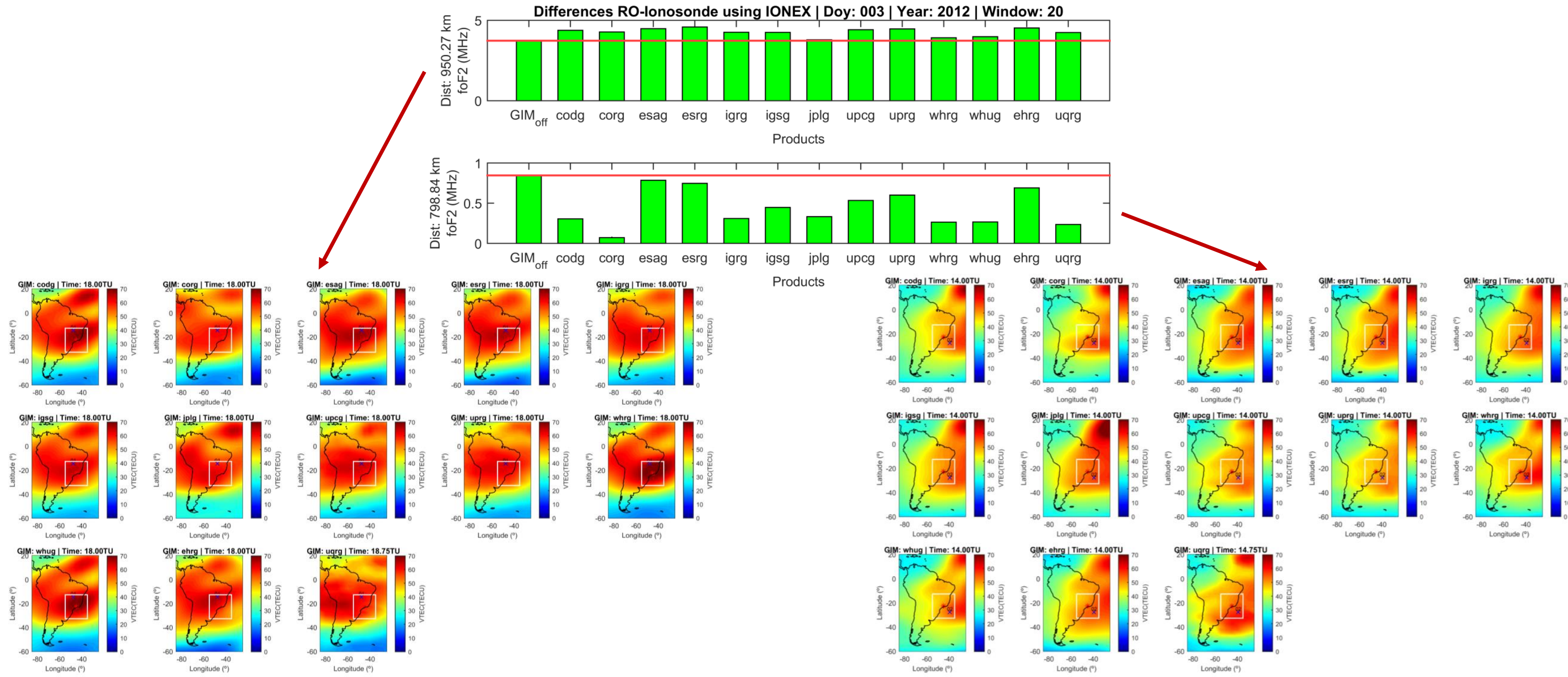


\section{Conclusions and future works}

- Significant part of the GNSS RO-derived electron density profiles presented better agreement of the critical frequency foF 2 with the direct ionosonde measurements results when GIM VTEC information is considered;

- Some dependency with the ionospheric intensity could be noticed, as well as dependency with the distance between the ionosonde and the occultation occurrence (in some cases);

- Some results did not present the same pattern, considering distance, region or ionospheric variability.

- Further investigation of the method considering the VTEC variability relation with the squared frequency, and the influence of the larger RO inversion error due to the larger variability of electron density over Brazil, should be performed in the next steps of this research;

- We also intend to expand the analysis to more days and other RO missions. 


\section{Thank you!}

gabriel.jerez@unesp.br

\section{unesp}

